Article

\title{
Association of TP53 Alteration with Tissue Specificity and Patient Outcome of IDH1-Mutant Glioma
}

\author{
Balazs Murnyak ${ }^{1}$ (D) and L. Eric Huang ${ }^{1,2, *(D)}$ \\ 1 Department of Neurosurgery, Clinical Neurosciences Center, University of Utah, \\ Salt Lake City, UT 84132, USA; balazs.murnyak@utah.edu \\ 2 Department of Oncological Sciences, Huntsman Cancer Institute, University of Utah, \\ Salt Lake City, UT 84112, USA \\ * Correspondence: eric.huang@hsc.utah.edu
}

check for updates

Citation: Murnyak, B.; Huang, L.E. Association of TP53 Alteration with Tissue Specificity and Patient Outcome of IDH1-Mutant Glioma. Cells 2021, 10, 2116. https://doi.org/ $10.3390 /$ cells 10082116

Academic Editors: Chun Zhang Yang and Prashant Chittiboina

Received: 26 July 2021

Accepted: 15 August 2021

Published: 17 August 2021

Publisher's Note: MDPI stays neutral with regard to jurisdictional claims in published maps and institutional affiliations.

Copyright: (c) 2021 by the authors. Licensee MDPI, Basel, Switzerland. This article is an open access article distributed under the terms and conditions of the Creative Commons Attribution (CC BY) license (https:// creativecommons.org/licenses/by/ $4.0 /)$.

\begin{abstract}
Since the initial discovery of recurrent isocitrate dehydrogenase 1 (IDH1) mutations at Arg132 in glioma, IDH1 hotspot mutations have been identified in cholangiocarcinoma, chondrosarcoma, leukemia, and various other types of cancer of sporadic incidence. Studies in glioma and leukemia have helped promote the theory that $I D H 1$ mutations are an oncogenic event that drives tumorigenesis in general. Through bioinformatic analysis of more than 45,000 human pan-cancer samples from three independent datasets, we show here that IDH1 mutations are rare events in human cancer but are exclusively prevalent in WHO grade II and grade III (lower-grade) glioma. Interestingly, alterations in the tumor-suppressor gene TP53 (tumor protein p53) co-occur significantly with IDH1 mutations and show a tendency of exclusivity to IDH2 mutations. The co-occurrence of IDH1 mutation and TP53 alteration is widespread in glioma, particularly in those harboring $I D H 1^{R 132 H}, I D H 1^{R 132 G}$, and $I D H 1^{R 132 S}$, whereas co-occurrence of $I D H 1^{R 132 C}$ and TP53 alteration can be found sporadically in other cancer types. In keeping with the importance of p53 in tumor suppression, TP53 status is an independent predictor of overall survival irrespective of histological and molecular subgroups in lower-grade glioma. Together, these results indicate tissue specificity of IDH1 hotspot mutation and TP53 alteration and the importance of TP53 status as a predictor of patient outcome in lower-grade glioma.
\end{abstract}

Keywords: glioma; IDH; isocitrate dehydrogenase; pan-cancer; patient outcome; survival; tissue specificity; TP53; tumor suppressor

\section{Introduction}

The IDH1 gene encodes $\mathrm{NADP}^{+}$-dependent IDH localized in the cytoplasm and peroxisomes [1-5]. This enzyme not only catalyzes the oxidative decarboxylation of isocitrate to 2-oxoglutarate but also is critical to reductive carboxylation, which is required for lipogenesis in hypoxia and redox homeostasis during anchorage-independent growth [6,7]. In agreement with its physiological function of regulating the intracellular $\mathrm{NADP}^{+} / \mathrm{NADPH}$ ratio [8], IDH1 plays an important role in metabolic adaption in physiology and cancer biology.

Earlier studies revealed widespread IDH1 mutations at Arg132, an active site of the enzyme, in WHO grade II and III (referred to as lower-grade) glioma and in WHO grade IV secondary glioblastoma [9-11]. Among these hotspot mutations, the $I D H 1^{R 132 H}$ frequency was $>90 \%$, whereas non-canonical IDH1 mutations, including IDH1 ${ }^{R 132 C}, I D H 1^{R 132 G}, I D H 1^{R 132 S}$, and $I D H 1^{R 132 L}$ (referred to collectively as $I D H 1^{R 132 X}$ ), were at very low frequencies [12]. Furthermore, mutations in the IDH2 gene (encoding a mitochondrial NADP ${ }^{+}$-dependent enzyme) at the analogous Arg172 were relatively uncommon in lower-grade glioma and non-existent in glioblastoma [13]. Although they were thought to be virtually exclusive in glioma [11,14], further mutational analyses revealed IDH1 and IDH2 mutations, as well as IDH2 Arg140 mutations, in various cancer types such as myeloid neoplasia, chondrosarcoma, cholangiocarcinoma, and prostate cancer $[2,15]$. 
IDH1 and IDH2 mutations acquire a neomorphic function that produces (D)-2-hydroxyglutarate (D-2HG) from the NADPH-dependent reduction of 2-oxoglutarate [16]. High levels of D-2HG induce histone and DNA hypermethylation through competitive inhibition of histone and DNA demethylases, thereby blocking cell differentiation and promoting neural stem-like phenotype [17-19]; however, neural stem-cell marker genes such as NES and PROM1 are generally downregulated in IDH-mutant glioma [20]. The finding that IDH1 and IDH2 mutations occur in various other cancer types has spurred further interest in cancer metabolism, epigenetic regulation, and therapeutic targeting, also promoting the idea that these mutations drive tumorigenesis in general [1-5,21], despite how IDH1 and IDH2 mutations promote gliomagenesis remains unclear [13,22].

Genetically, lower-grade gliomas with IDH1 and IDH2 mutations are associated with either TP53 and/or ATRX alteration or $1 \mathrm{p} / 19 \mathrm{q}$ codeletion $[23,24]$. Although TP53 is among the most-mutated tumor-suppressor genes in human cancer [25-27], the biological significance of TP53 alteration in lower-grade glioma requires further investigation. In this study, we analyzed three independent pan-cancer datasets and revealed that the association of IDH1 mutation with TP53 alteration is specific to glioma, which indicates a tissue-specific role for TP53 alteration in gliomagenesis.

\section{Materials and Methods}

\subsection{Pan-Cancer Datasets}

Three independent pan-cancer datasets: TCGA PanCancer dataset (TCGA_PanCancer); MSK-Impact pan-cancer dataset (MSK_Impact); and a combined, non-redundant pancancer dataset (Non-Redundant), were downloaded from cBioPortal [28,29]. Downloaded data included study ID, sample ID, patient ID, and patient status and survival, with matched genetic alteration data of IDH1, IDH2, TP53, CDKN2A, CDKN2B, CIC, FUBP1, and $1 p / 19 q$ codeletion.

TCGA_PanCancer consists of 32 studies comprising 10,953 patients /10,967 samples from cancer types including bladder urothelial carcinoma (BLCA, $n=411$ ), cholangiocarcinoma (CHOL, $n=36$ ), colorectal adenocarcinoma (COADREAD, $n=594$ ), breast invasive carcinoma (BRCA, $n=1084$ ), brain lower-grade glioma (LGG, $n=514$ ), glioblastoma (GBM, $n=592$ ), esophageal adenocarcinoma (ESCA, $n=182$ ), stomach adenocarcinoma (STAD, $n=440$ ), head and neck squamous cell carcinoma (HNSC, $n=523$ ), liver hepatocellular carcinoma (LIHC, $n=372$ ), lung adenocarcinoma (LUAD, $n=566$ ), lung squamous cell carcinoma (LUSC, $n=487$ ), acute myeloid leukemia (LAML, $n=200$ ), ovarian serous cystadenocarcinoma $(\mathrm{OV}, n=585)$, pancreatic adenocarcinoma (PAAD, $n=184)$, skin cutaneous melanoma (SKCM, $n=448$ ), sarcoma (SARC, $n=255$ ), and uterine corpus endometrial carcinoma (UCEC, $n=529)$.

MSK_Impact consists of 10,336 patients /10,945 profiled samples, including glioma ( $n=553)$, hepatobiliary cancer (Hepatobiliary, $n=355)$, bone cancer (Bone, $n=135$ ), skin cancer, non-melanoma (SKNM, $n=148$ ), small cell lung cancer (SCLC, $n=82$ ), melanoma $(n=365)$, mature $\mathrm{T}$ and NK neoplasms (Mature $\mathrm{T}$ and $\mathrm{NK}, n=134)$, uterine sarcoma (USARC, $n=93$ ), small bowel cancer (Small Bowel, $n=35$ ), and central nervous system cancer (CNS, $n=48)$.

Non-Redundant consists of 152 studies published from numerous institutions comprising 25,016 patients /26,922 samples with various cancer types, including acute myeloid leukemia or myelodysplastic syndromes ( $\mathrm{mnm})$, skin cutaneous melanoma ( $\mathrm{skcm})$, metastatic melanoma (mel), uterine carcinoma (ucs), cutaneous squamous cell carcinoma (cscc), primary central nervous system lymphoma (pcnsl), esophageal squamous cell carcinoma (escc), esophageal adenocarcinoma (esca), ampullary carcinoma (ampca), and basal cell carcinoma (bcc).

\subsection{Data Analysis}

Relevant data were extracted and processed with GraphPad Prism version 9.0 software (GraphPad Software, San Diego, CA, USA) to present genetic alteration frequency and 
occurrence. Statistical significance in frequency difference was determined by paired $t$ test or Fisher's exact test, as specified, with two-tailed $p$-values. Overall survival was analyzed by the Mantel-Cox log-rank test as previously described [30,31]. Multivariate Cox proportional hazards analysis was performed with SPSS Statistics (IBM) software by including TP53 status, IDH1 status, age, sex, and histological type, as previously described [31].

\section{Results}

\subsection{IDH1 Hotspot Mutations Are a Rare Event but Prevalent Exclusively in Lower-Grade Glioma}

To obtain a landscape of IDH1 and IDH2 mutations in human cancer, we analyzed samples from three independent pan-cancer datasets: TCGA PanCancer dataset (TCGA_PanCancer); MSK-Impact pan-cancer dataset (MSK_Impact); and a combined, non-redundant pancancer dataset (Non-Redundant), which comprised a total of 45,228 human samples with various cancer types (see Materials and Methods).

Analysis of TCGA_PanCancer revealed overall frequencies of IDH1 and IDH2 alterations, including mutation, homozygous deletion, and amplification, at $6 \%$ and $2 \%$, respectively. Among these alterations, IDH1 hotspot mutations were $<5 \%$ (or 480 ) and isolated alterations were $<1 \%$, whereas nearly $95 \%$ samples had no alteration (Figure 1 ; Table 1). Likewise, $99 \%$ of samples showed no alteration in $I D H 2$, and only $0.4 \%$ (or 46 ) samples had Arg140 or Arg172 mutations (Table S1). The low frequencies of IDH1 and IDH2 mutations in human cancer were confirmed with MSK_Impact; the overall frequencies of IDH1 and IDH2 alterations were $3 \%$ and $<1 \%$, respectively; and IDH1 and IDH2 hotspot mutations were $2 \%$ and $0.3 \%$ (or 260 and 31), respectively (Figure 1; Table 1 and Table S1). Moreover, similar results were obtained from Non-Redundant, with IDH1 and IDH 2 hotspot mutations at $1 \%$ and $0.4 \%$, respectively (Figure 1 ; Table 1 and Table S1). Thus, the overall frequencies of IDH1 and IDH2 hotspot mutations were $2 \%$ and $0.4 \%$, respectively. In contrast to the high frequencies of TP53 alteration averaging 32\% (Figure 1), these results indicate that both IDH1 and IDH2 hotspot mutations are rare events in human cancer.

Table 1. Occurrence and frequency of IDH1 alteration in human cancer.

\begin{tabular}{cccccccc}
\hline Dataset & Samples & \multicolumn{2}{c}{ R132 } & \multicolumn{2}{c}{ Isolated } & \multicolumn{2}{c}{ No Alteration } \\
\hline TCGA_PanCancer & 10,439 & 480 & $5 \%$ & 74 & $1 \%$ & 9884 & $95 \%$ \\
MSK_Impact & 10,945 & 260 & $2 \%$ & 74 & $1 \%$ & 10,610 & $97 \%$ \\
Non-Redundant & 23,844 & 219 & $1 \%$ & 148 & $1 \%$ & 23,256 & $98 \%$ \\
\hline Total & 45,228 & 961 & $2 \%$ & 296 & $1 \%$ & 43,750 & $97 \%$ \\
\hline
\end{tabular}

The frequency of IDH1 mutation in lower-grade glioma, however, was conspicuously high $(77 \%)$ in TCGA_PanCancer, with cholangiocarcinoma and acute myeloid leukemia much lower at $14 \%$ and $<10 \%$, respectively (Figure $2 \mathrm{~A}$ ). IDH2 mutation was most common in acute myeloid leukemia (11\%), followed by $<6 \%$ in cholangiocarcinoma; however, IDH2 amplification was more common, albeit at low frequencies, among various cancer types (Figure 2B). In MSK_Impact, IDH1 alteration was 33\% in various types of gliomas and $14 \%$ in hepatobiliary cancer (Figure 2D), whereas IDH2 mutation was seen most frequently in mature T and NK neoplasms (Figure 2E). In Non-Redundant, the frequency of IDH1 mutation was $100 \%$ in lower-grade glioma, and cancer types with IDH2 mutation $>10 \%$ included acute myeloid leukemia, myelodysplastic syndromes, and primary central nervous system lymphoma (Figure S1A,B). Again, IDH2 amplification was seen particularly in prostate adenocarcinoma, pancreatic adenocarcinoma, melanoma, and invasive breast carcinoma. In contrast, TP53 mutation was widespread among various cancer types (Figure 2C,F and Figure S1C). Therefore, although both IDH1 and IDH2 mutations are rare events in human cancer, the prevalence of IDH1 mutation in lower-grade glioma suggests a tissue-specific role in tumorigenesis. 


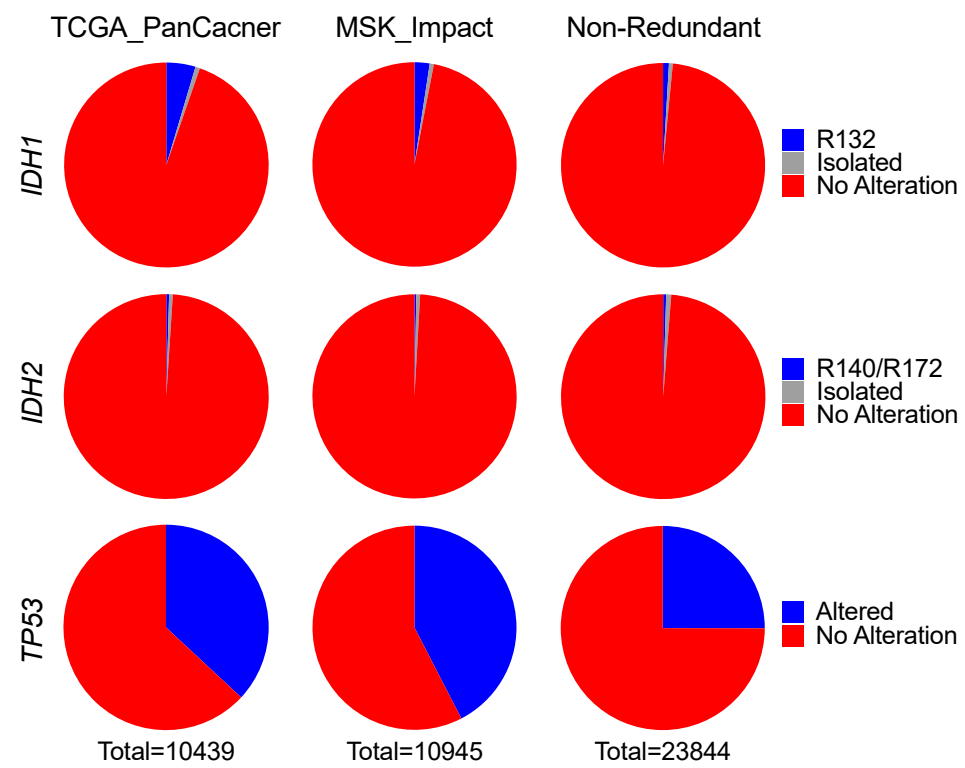

Figure 1. IDH1 and IDH2 alterations are rare in human cancer. Recurrent Arg132 mutation (R132) in IDH1, isolated genetic events (isolated), and no alteration were extracted from the TCGA_PanCancer, MSK_Impact, and Non-Redundant datasets. IDH2 Arg140 and Arg172 (R140/R172) mutations and TP53 alterations were analyzed similarly.
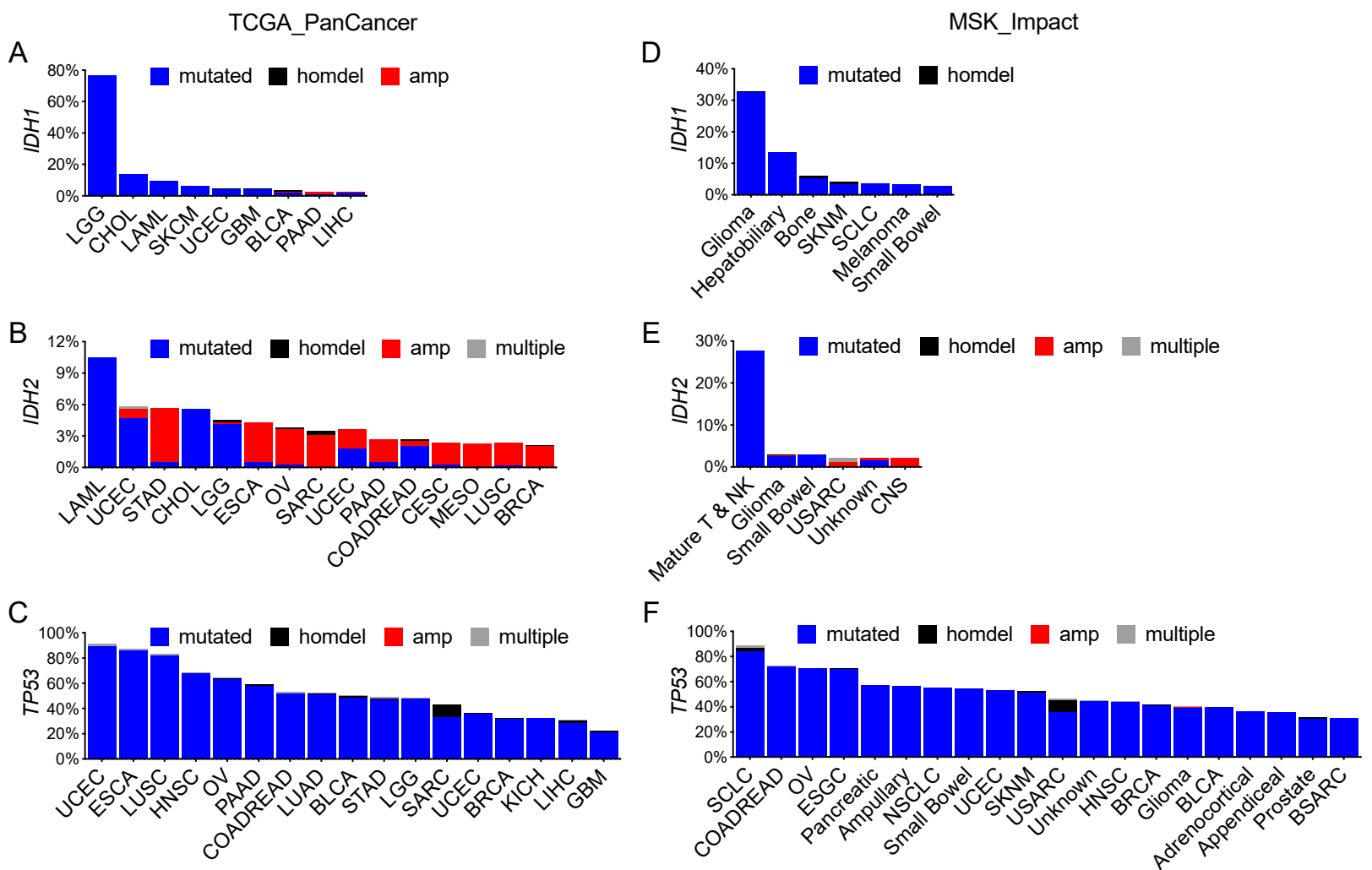

Figure 2. Distinctive distribution of $I D H 1, I D H 2$, and TP53 alterations in human cancer. Extraction of IDH1, IDH2, and TP53 alterations from specified datasets revealed a high frequency of IDH1 mutations exclusively in glioma (A,D). Whereas relatively high frequencies of $I D H 2$ mutation were limited to hematopoietic neoplasms, IDH2 amplification (amp) was seen in more cancer types (B,E) and TP53 alteration was widespread (C,F). The cutoff is $2 \%$ for IDH1 and IDH2 and $20 \%(\mathbf{C})$ or $30 \%$ (F) for TP53. 


\subsection{Co-Occurrence of IDH1 Hotspot Mutation and TP53 Alteration Predominantly in Glioma}

Despite the low frequency of IDH1 mutation in human cancer, further analysis revealed significant to extremely significant co-occurrence of IDH1 and TP53 alterations but mutual exclusivity between IDH2 and TP53 alterations in all three datasets (Table 2 and Table S2). Furthermore, the overall frequency of IDH1 hotspot mutation co-occurring with TP53 alteration was $49 \%$ versus $23 \%$ for the co-occurrence of $I D H 1$ isolated alteration and TP53 alteration (Table 3). Specifically, the co-occurrence frequency remained above $50 \%$ for $I D H 1^{R 132 H}$, IDH1 $1^{R 132 G}$, and $I D H 1^{R 132 S}$, but much lower for $I D H 1^{R 132 C}$ and $I D H 1^{R 132 L}$ (Table 4). Consistent with the mutual exclusivity, only $6 \%(11 / 182)$ of IDH2 hotspot mutations co-occurred with TP53 alteration (Table S3). These results indicate that TP53 alterations exhibit a tendency of co-occurring with IDH1, but not IDH2, mutations in human cancer.

Table 2. Co-occurrence of IDH1 and TP53 alterations in human cancer.

\begin{tabular}{ccccccccc}
\hline Dataset & IDH1 & TP53 & Both & Neither & Log2 OR & $\boldsymbol{p}$-Value & $\boldsymbol{q}$-Value & Tendency \\
\hline TCGA_PanCancer & 256 & 3557 & 299 & 6327 & 1.055 & $<0.001$ & $<0.001$ & Co-occurrence \\
MSK_Impact & 173 & 4460 & 161 & 6151 & 0.360 & 0.014 & 0.043 & Co-occurrence \\
Non-Redundant & 203 & 4766 & 143 & 12,865 & 0.927 & $<0.001$ & $<0.001$ & Co-occurrence \\
\hline
\end{tabular}

Table 3. Co-occurrence frequencies of TP53 alteration and IDH1 hotspot mutation or isolated alteration in human cancer.

\begin{tabular}{cccccc}
\hline Dataset & \multicolumn{2}{c}{ R132 } & Isolated & $\begin{array}{c}\text { Fisher's Exact } \\
p \text {-Value }\end{array}$ \\
\hline TCGA_PanCancer & $264 / 480$ & $55 \%$ & $118 / 491$ & $24 \%$ & $<0.0001$ \\
MSK_Impact & $117 / 260$ & $45 \%$ & $45 / 74$ & $61 \%$ & 0.0179 \\
Non-Redundant & $89 / 217$ & $41 \%$ & $1782 / 7819$ & $23 \%$ & $<0.0001$ \\
\hline Total & $470 / 957$ & $49 \%$ & $1945 / 8384$ & $23 \%$ & $<0.0001$ \\
\hline
\end{tabular}

Table 4. Co-occurrence frequencies of specific IDH1-R132 mutation and TP53 alteration in human cancer.

\begin{tabular}{ccccccc}
\hline Dataset & R132H & R132C & R132G & R132S & R132L & R132I \\
\hline TCGA_PanCancer & $220 / 389(57 \%)$ & $21 / 61(34 \%)$ & $13 / 16(81 \%)$ & $9 / 11(82 \%)$ & $1 / 3(33 \%)$ & \\
MSK_Impact & $90 / 168(54 \%)$ & $17 / 70(24 \%)$ & $5 / 9(56 \%)$ & $3 / 4(75 \%)$ & $2 / 8(25 \%)$ & $0 / 1(0 \%)$ \\
Non-Redundant & $74 / 115(64 \%)$ & $10 / 77(13 \%)$ & $5 / 9(56 \%)$ & $0 / 9(0 \%)$ & $0 / 7(0 \%)$ & $0 / 1(0 \%)$ \\
\hline Total & $384 / 672(57 \%)$ & $48 / 208(23 \%)$ & $23 / 34(68 \%)$ & $12 / 24(50 \%)$ & $3 / 18(17 \%)$ & 0 \\
\hline
\end{tabular}

To assess whether such co-occurrence is cancer-type specific, we extracted all cancer types harboring IDH1 hotspot mutation and TP53 alteration. Interestingly, 97\% of the co-occurrences were in lower-grade glioma and glioblastoma in TCGA_PanCancer, with the rest including melanoma and lung adenocarcinoma (Figure 3A; Table S4). In particular, $I D H 1^{R 132 H}, I D H 1^{R 132 G}$, and $I D H 1^{R 132 S}$ co-occurrences were exclusive to glioma, whereas $I D H 1^{R 132 C}$ co-occurrence was seen in various cancer types (Figure 3B; Table S4). In MSK_Impact, $87 \%$ of the co-occurrences were gliomas of various types, and the rest included cholangiocarcinoma, lung adenocarcinoma, and chondrosarcoma (Figure 3C; Table S5). Again, $I D H 1^{R 132 H}, I D H 1^{R 132 G}$, and $I D H 1^{R 132 S}$ co-occurrences were virtually exclusive to glioma except for single cases of $I D H 1^{R 132 H}$ astroblastoma, $I D H 1^{R 132 H}$ adenoid cystic carcinoma, and $I D H 1^{R 132 G}$ lung adenocarcinoma (Figure 3D; Table S5). Lastly, in Non-Redundant, gliomas of various types accounted for $90 \%$ of the co-occurrences, whereas acute myeloid leukemias were only $4 \%$ (Figure 3E; Table S6). Specifically, $96 \%$ of the $I D H 1^{R 132 H}$ co-occurrences and $60 \%$ of the $I D H 1^{R 132 G}$ co-occurrences were in glioma (Figure 3F; Table S6). As expected, co-occurrences of IDH2 hotspot mutations and TP53 alterations were extremely rare; there were a total of 11 cases among all three datasets, 
including 4 cases of lower-grade glioma, 3 cases of acute myeloid leukemia, and 2 cases of basal cell carcinoma (Figure S2). Therefore, the virtual exclusivity of co-occurrence of IDH1 hotspot mutation and TP53 alteration in glioma indicates the importance of TP53 alteration in IDH1-mutant gliomagenesis.
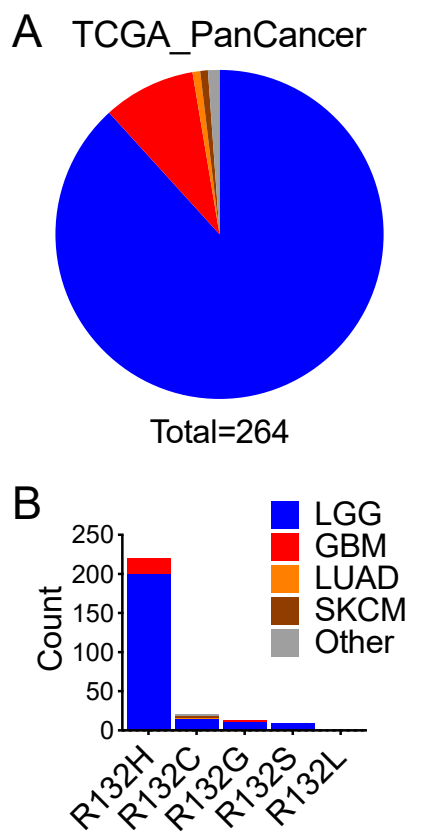
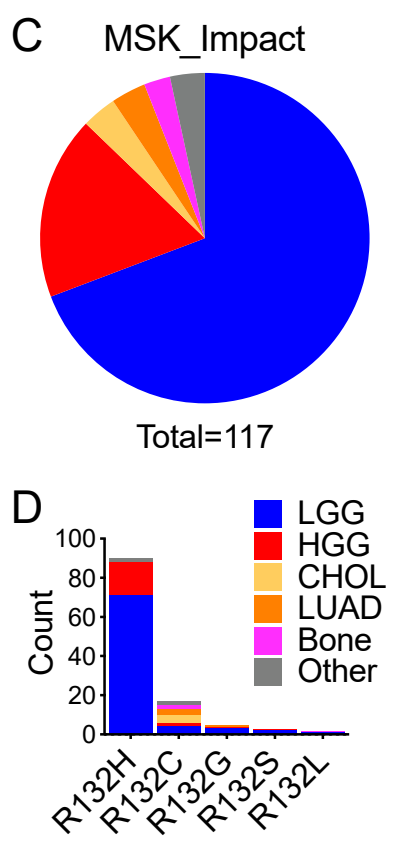
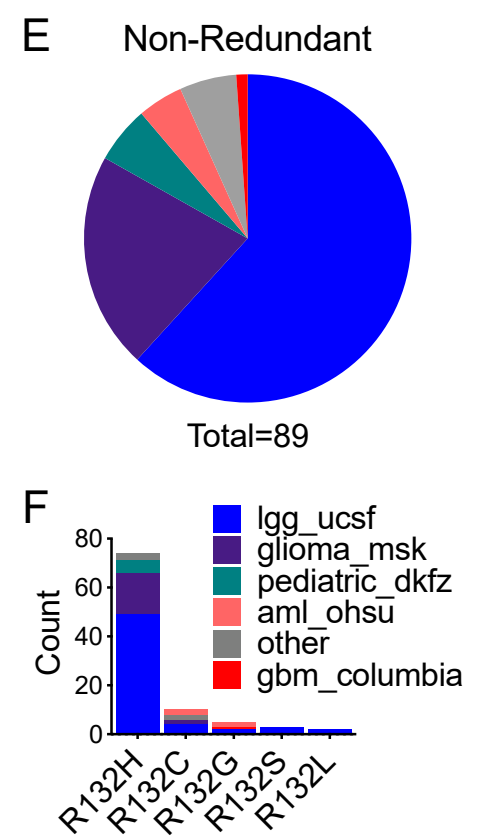

Figure 3. Co-occurrence of IDH1 hotspot mutation and TP53 alteration is predominantly in glioma. Analysis of TCGA_PanCancer (A,B), MSK_Impact (C,D), and Non-Redundant (E,F) datasets reveals co-occurrence of IDH1 hotspot mutations and TP53 alterations overwhelmingly in glioma and rarely in other cancer types, as presented in pie charts (top) and column charts (bottom) where sample counts of the cancer types are in reference to specific types of IDH1 mutation. Of note, the cancer types in pediatric_dkfz $(\mathbf{F})$ are high-grade glioma.

\subsection{Differential Co-Occurrence Frequencies between IDH1 ${ }^{R 132 H}$ and IDH1 $1^{\text {R132X }}$ in Glioma}

Non-canonical IDH1 ${ }^{R 132 X}$ occurs in $8 \%$ of lower-grade glioma harboring IDH1 hotspot mutations [13]. In keeping with the notion that co-occurrence of IDH1 hotspot mutation and TP53 alteration is glioma-specific, the mean co-occurrence frequency was fivefold greater in glioma than in non-glioma (Figure $4 \mathrm{~A}$ ); however, the difference in $I D H 1^{R 132 H}$ co-occurrence frequencies between glioma and non-glioma was not statistically significant (Figure 4B), even though $I D H 1^{R 132 H}$ occurred in $92 \%$ in lower-grade glioma harboring IDH1 hotspot mutations [13]. In contrast, whereas $I D H 1^{R 132 C}$ is the major form in chondrosarcoma, cholangiocarcinoma, and acute myeloid leukemia [13], the co-occurrence of $I D H 1^{R 132 C}$ and TP53 alteration was nearly eightfold greater in glioma compared with non-glioma (Figure S3), as was the co-occurrence of combined IDH $1^{R 132 X}$ (Figure 4B).

The significant co-occurrence of IDH1 hotspot mutation and TP53 alteration in glioma was in accordance with the consistently high frequencies found across various histological subtypes, including glioblastoma, from both TCGA_PanCancer and MSK_Impact (Table 5). In contrast, the co-occurrence frequency of $I D H 1^{R 132 H}$ and TP53 alteration in oligodendroglioma averaged $17 \%$ versus $>90 \%$ in astrocytoma and glioblastoma. Despite the rare occurrence of TP53 alteration in oligodendroglioma [24], the TP53 alteration frequency in $I D H 1^{R 132 X}$ oligodendroglioma was $100 \%(5 / 5)$, significantly greater than that of $I D H 1^{R 132 H}$ oligodendroglioma ( $p=0.0012$, Fisher's exact test). Of note, given the mutual exclusivity of TP53 alteration and 1p/19q codeletion in IDH-mutant lower-grade glioma [24], none of the $I D H 1^{R 132 X}$ oligodendrogliomas harbored CIC and/or FUBP1 mutations that are associated with $1 \mathrm{p} / 19 \mathrm{q}$ codeletion. 


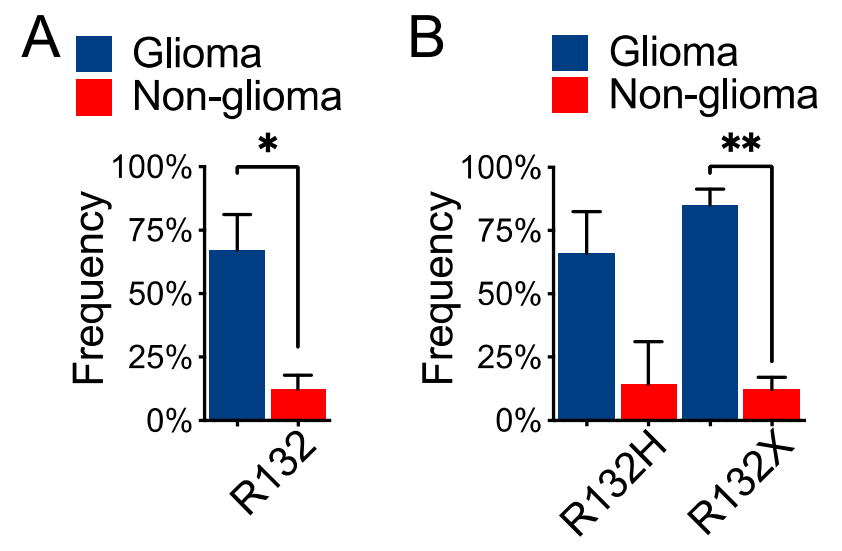

Figure 4. Higher frequencies of co-occurrence of IDH1 hotspot mutation and TP53 alteration in glioma. Glioma and non-glioma were compared for their co-occurrence frequencies of TP53 alteration and IDH1-R132 mutation (A), and TP53 alteration and IDH1 ${ }^{R 132 H}$ or IDH1 ${ }^{R 132 X}(\mathbf{B}){ }^{*} p<0.05$; $^{* *} p<0.01$.

Table 5. Co-occurrence frequencies of specific IDH1 hotspot mutation and TP53 alteration in different histological subtypes of glioma.

\begin{tabular}{ccccccc}
\hline \multirow{2}{*}{ Cancer Type } & \multicolumn{2}{c}{ TCGA_PanCancer } & \multicolumn{2}{c}{ MSK_Impact } & \multicolumn{2}{c}{ Combined } \\
\cline { 2 - 6 } & $\mathbf{R 1 3 2 H}$ & $\mathbf{R 1 3 2 X}$ & $\mathbf{R 1 3 2 H}$ & $\mathbf{R 1 3 2 X}$ & $\mathbf{R 1 3 2 H}$ & R132X \\
\hline Astrocytoma & $100 / 112(89 \%)$ & $19 / 21(90 \%)$ & $56 / 57(98 \%)$ & $9 / 9(100 \%)$ & $156 / 169(92 \%)$ & $28 / 30(93 \%)$ \\
Glioblastoma & $21 / 22(95 \%)$ & $3 / 3(100 \%)$ & $16 / 18(89 \%)$ & $3 / 3(100 \%)$ & $37 / 40(93 \%)$ & $6 / 6(100 \%)$ \\
Oligoastrocytoma & $64 / 98(65 \%)$ & $10 / 11(91 \%)$ & $11 / 16(69 \%)$ & $1 / 2(50 \%)$ & $75 / 114(66 \%)$ & $11 / 12(92 \%)$ \\
Oligodendroglioma & $33 / 147(22 \%)$ & $5 / 5(100 \%)$ & $3 / 67(4 \%)$ & NA & $36 / 214(17 \%)$ & $5 / 5(100 \%)$ \\
\hline Total & $218 / 379(58 \%)$ & $37 / 40(93 \%)$ & $86 / 158(54 \%)$ & $13 / 14(93 \%)$ & $304 / 537(57 \%)$ & $50 / 54(93 \%)$ \\
\hline
\end{tabular}

Co-occurrence of specific IDH1 mutation and TP53 alteration is expressed as a percentage of total count in each histological subtype of glioma.

\subsection{TP53 Status Is an Independent Predictor of Patient Survival in Lower-Grade Glioma}

Glioma patients with IDH1 hotspot mutations are known to have better survival than those without such mutations [11,24,32], but astrocytoma patients with non-canonical $I D H 1^{R 132 X}$ have even longer survival than those with $I D H 1^{R 132 H}$ [33]. By following the latest cIMPACT-NOW recommendation that IDH-mutant gliomas harboring homozygous $C D K N 2 A / B$ deletion are equivalent to IDH1-wildtype [34], we not only confirmed this finding in the TCGA-LGG dataset but, more importantly, observed the role of TP53 status in patient survival (Figure 5A,B). TP53 status distinguished survival in both $I D H 1^{R 132 H}$ and $I D H 1^{R 132 X}$ subgroups despite the significant increase in overall survival in patients with $I D H 1^{R 132 X}$ compared with those with $I D H 1^{R 132 H}$. The clustering of TP53-wildtype $I D H 1^{R 132 H}$ glioma and TP53-altered $I D H 1^{R 132 X}$ glioma in overall survival underscored the paramount importance of TP53 status in the outcomes of glioma patients. Moreover, similar significant associations were observed in the entire cohort and in histological and molecular subgroups including oligodendroglioma and IDH1-wildtype glioma (Figure 5C and Figure S4), in agreement with the tumor-suppressive function of p53 in human cancer $[27,35,36]$.

To confirm these results, we performed a multivariate Cox proportional hazards analysis and found that TP53 status was significant in the IDH1 hotspot mutation subgroup (HR $=2.079 ; 95 \% \mathrm{CI}: 1.083-3.992 ; p=0.028)$, in the oligodendroglioma subgroup $(\mathrm{HR}=2.001 ; 95 \%$ CI: $1.032-3.879 ; p=0.040)$ (Tables 6 and 7), as well as in the entire cohort $(\mathrm{HR}=1.809 ; 95 \% \mathrm{CI}: 1.327-3.150 ; p=0.001)$ and the IDH1-wildtype subgroup (HR = 2.572; 95\% CI: 1.378-4.802; $p=0.003$ ) (Tables S7 and S8). Therefore, TP53 status is an independent predictor of patient survival in lower-grade glioma irrespective of molecular and histological subclassifications. 

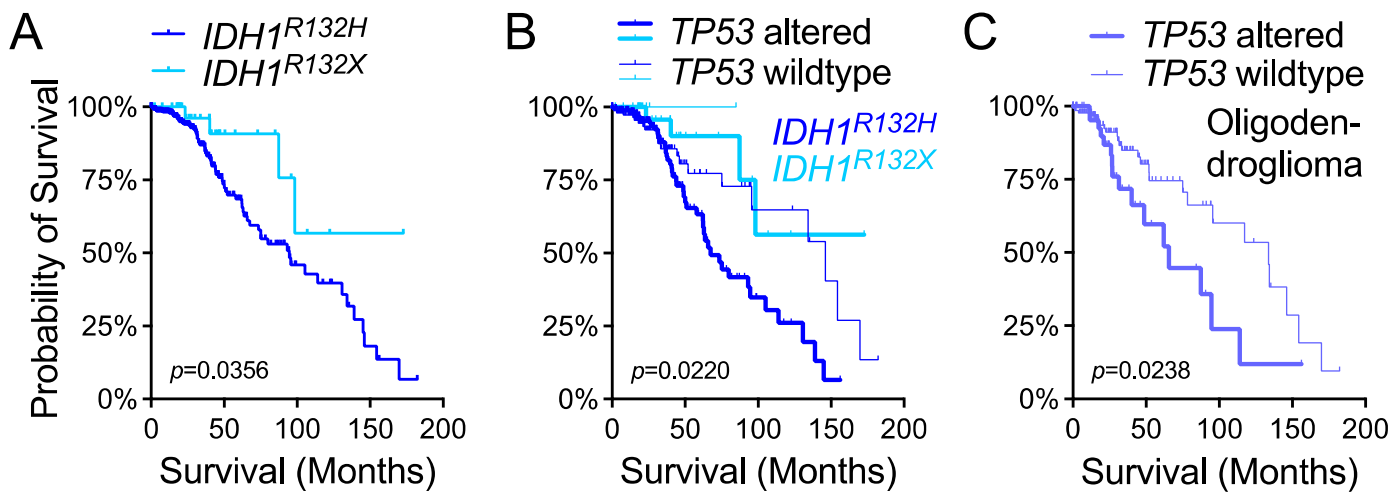

Figure 5. TP53 status determines patient survival outcomes in molecular and histological subgroups of lower-grade glioma in TCGA_PanCancer. Differences in overall survival were analyzed between $I D H 1^{R 132 H}$ and $I D H 1^{R 132 X}$ subgroups (A) and among those of TP53 wildtype (thin line) and TP53 altered (thick line) (B). Overall survival was also analyzed in the oligodendroglioma subgroup with respect to TP53 status (C). Two-tailed $p$-values are specified.

Table 6. Multivariate Cox proportional hazards analysis of TP53 status in the IDH1 hotspot mutation subgroup of TCGA-LGG dataset.

\begin{tabular}{ccccc}
\hline & Hazards Ratio & \multicolumn{2}{c}{$\mathbf{9 5 \%}$ CI } & $p$-Value \\
\hline TP53 no alteration vs. altered & 2.079 & 1.083 & 3.992 & 0.028 \\
IDH1 R132H vs. R132X & 0.348 & 0.122 & 0.991 & 0.048 \\
Age <40 vs. >60 years old & 4.649 & 2.25 & 9.608 & $<0.001$ \\
Male vs. Female & 1.048 & 0.644 & 1.705 & 0.85 \\
Oligodendroglioma vs. Astrocytoma & 1.085 & 0.546 & 2.158 & 0.815 \\
\hline
\end{tabular}

Table 7. Multivariate Cox proportional hazards analysis of TP53 status in the oligodendroglioma subgroup of TCGA-LGG dataset.

\begin{tabular}{ccccc}
\hline & Hazards Ratio & \multicolumn{2}{c}{$\mathbf{9 5 \%}$ CI } & $p$ Value \\
\hline TP53 no alteration vs. altered & 2.001 & 1.032 & 3.879 & 0.040 \\
IDH1 wildtype vs. R132 & 0.692 & 0.485 & 0.986 & 0.042 \\
Age <40 vs. >60 years old & 11.696 & 4.409 & 31.026 & $<0.001$ \\
Male vs. Female & 0.787 & 0.401 & 1.545 & 0.486 \\
\hline
\end{tabular}

\section{Discussion}

Through a survey of more than 45,000 pan-cancer samples, we observed that IDH1 and $I D H 2$ hotspot mutations are uncommon (2\%) and extremely rare $(0.4 \%)$, respectively, in human cancer, a finding in agreement with an independent pan-cancer analysis [37]. Therefore, despite being prevalent in glioma, as reported previously $[10,11,14,38]$, these mutations appear to be selected against in tumorigenesis, which is seemingly at odds with the general thought that these mutations induce oncogenic transformation through epigenetic and metabolic reprogramming resulting from high levels of D-2HG [13]. IDH1 ${ }^{\text {R132H }}$, the most common form in glioma, produces the least amount of D-2HG and correlates with worse survival compared with the rare IDH1 ${ }^{R 132 X}$ and IDH2-R172 mutations, which produce higher levels of D-2HG [12,33,39]. Although the rare occurrence of these mutations in glioma has been attributable to the "cytotoxicity" of high levels of D-2HG [12,33], D-2HG sensitizes cells to ferroptosis [40] -an iron-dependent form of nonapoptotic cell death likely involved in tumor suppression [41]. D-2HG also exhibits tumor-suppressive activities through the inhibition of aerobic glycolysis in both IDH-mutant and IDH-wildtype leukemia cells [42]. Together with our previous studies showing that IDH1 hotspot mutations are intrinsically tumor suppressive $[30,43,44]$, these findings may provide an explanation for the rare occurrence of IDH1 and IDH2 hotspot mutations in human cancer. 
The prevalence of IDH1 hotspot mutation in glioma and its co-occurrence with TP53 alteration indicate a tissue-specific role in gliomagenesis [13]. Tissue specificity in cancer is best evidenced by hereditary cancer predisposition syndromes in which the underlying gene defects, such as mutations in $A P C, B R C A 1$, and $V H L$, are associated with a high risk of developing tissue-specific cancer types [45]. In nonhereditary cancers, a subset of recurring genetic alterations can be identified to be associated with a particular type of cancer $[45,46]$. What drives tissue specificity in cancer, however, is complex even though numerous possibilities, including cell of origin, heterogeneity, epigenetic state, and environment, have been proposed $[45,46]$. In keeping with this, studies have shown the requirement of Trp53 knockout/down to recapitulate a less aggressive phenotype of $I D H 1^{R 132 H}$ glioma compared with IDH1-wildtype glioma [47-49]; however, the mechanism by which TP53 alteration contributes to gliomagenesis remains unclear.

Interestingly, p53-mediated ferroptosis, a novel function of p53, has been implicated in tumor suppression independent of its previously recognized tumor-suppressive activities in cell cycle, apoptosis, and senescence [50-54]. Given the strongest display of ferroptosissensitive gene signature in IDH-mutant lower-grade glioma among all cancer types [55], we speculate that TP53 alteration is required to inhibit ferroptosis for gliomagenesis, especially for $I D H 1^{R 132 X}$ gliomas, including oligodendroglioma, that are supersensitive to ferroptosis owing to the higher levels of D-2HG. For $I D H 1^{R 132 H}$ gliomas that are relatively less sensitive to ferroptosis, alternative tumor-suppressor pathways, such as $1 \mathrm{p} / 19 \mathrm{q}$ codeletion, must be inactivated. Furthermore, our previous studies suggested the importance of the glutamaterich cerebral environment in IDH-mutant lower-grade gliomagenesis [44,56], in agreement with the role of environment for tissue specificity in cancer. Therefore, the requirement of TP53 alteration and a glutamate-rich environment in gliomagenesis warrants further investigation to account for the prevalence of IDH1 hotspot mutations in glioma.

The tendency of mutual exclusivity between IDH2 and TP53 alteration in human cancer, including glioma, is intriguing, which may suggest alternative mechanisms of tumor-suppressor gene inactivation in tumorigenesis. Given the higher levels of D-2HG and its association with better survival [33,39], understanding how IDH2-R172 glioma cells overcome D-2HG induced sensitization to ferroptosis will shed light on the mechanism of $\mathrm{IDH} 2$-mutant gliomagenesis and the rare occurrence of such a mutation in human cancer.

Although the p53 tumor-suppressor pathway is altered at the frequency of $87 \%$ in glioblastoma [57], previously, the TP53 status has not been associated with patient survival outcomes despite the well-established association of TP53 alteration with IDH mutations in glioma $[23,24,58,59]$. Interestingly, TP53 alteration has been associated with poor outcomes in pediatric H3 K27M-mutant glioma [60]. Likewise, in lower-grade glioma we provided evidence that TP53 status is an independent predictor of overall survival in various molecular and histological subgroups, including the $I D H 1^{R 132 X}$ subgroup, which was recently reported to have better outcomes than the $I D H 1^{R 132 H}$ subgroup [33]. Interestingly, we observed similar overall survival between the TP53-wildtype $I D H 1^{R 132 H}$ subgroup and the TP53-altered IDH1 ${ }^{R 132 X}$ subgroup based on the available size of samples; however, the paramount importance of TP53 status in $I D H 1^{R 132 X}$ glioma requires validation with independent cohorts. Similarly, the importance of TP53 status in oligodendroglioma patient survival also requires validation. Moreover, additional genetic events, such as ATRX, $T E R T$, and $B R A F$, which also frequently occur in IDH-mutant glioma $[24,61,62]$, should be considered in future investigations.

\section{Conclusions}

IDH1 hotspot mutations are rare events in human cancer but prevalent in glioma. The co-occurrence of IDH1 hotspot mutation and TP53 alteration indicates the tissue specificity of these genetic changes in gliomagenesis. TP53 status is an important predictor of overall survival in lower-grade glioma. 
Supplementary Materials: The following are available online at https: / www.mdpi.com/article/ 10.3390/ cells10082116/s1, Table S1: Occurrence and frequency of IDH2 alteration in human cancer, Table S2: Mutual exclusivity of IDH2 and TP53 alterations in human cancer, Table S3: Low cooccurrence frequencies of specific IDH2 mutation and TP53 alteration in human cancer, Table S4: Gliomas are the predominant cancer type harboring co-occurrence of specific IDH1 mutation and TP53 alteration in TCGA_PanCancer, Table S5: Gliomas of various histological types constitute the predominant cancer type harboring co-occurrence of specific IDH1 mutation and TP53 alteration in MSK_Impact, Table S6: Gliomas of different studies collectively constitute the predominant cancer type harboring co-occurrence of specific IDH1 mutation and TP53 alteration in Non-Redundant, Table S7: Multivariate Cox proportional hazards analysis of TP53 status in TCGA-LGG dataset, Table S8: Multivariate Cox proportional hazards analysis of TP53 status in the IDH1-wildtype subgroup of TCGA-LGG dataset, Figure S1: Distinctive distribution of IDH1, IDH2, and TP53 alterations in Non-Redundant, Figure S2: Rare co-occurrence of IDH2 hotspot mutation and TP53 alteration in human cancer, Figure S3: Higher frequencies of co-occurrence of specific IDH1 mutation and TP53 alteration in glioma, Figure S4: IDH1 status and TP53 status distinguish patient survival in lower-grade glioma of TCGA_PanCancer.

Author Contributions: Conceptualization, L.E.H.; methodology, L.E.H. and B.M.; validation, L.E.H. and B.M.; formal analysis, L.E.H. and B.M.; data curation, L.E.H. and B.M.; writing-original draft preparation, L.E.H. and B.M.; writing-review and editing, L.E.H. and B.M.; visualization, L.E.H.; supervision, L.E.H.; project administration, L.E.H.; funding acquisition, L.E.H. All authors have read and agreed to the published version of the manuscript.

Funding: This work was supported in part by the National Institute of Neurological Disorders and Stroke (1R21NS108065) and the Department of Neurosurgery at the University of Utah.

Institutional Review Board Statement: Ethical review and approval were waived for this study, due to the use of only publicly available human datasets. No patient was recruited specifically for this study.

Informed Consent Statement: Not applicable.

Data Availability Statement: Available upon request.

Acknowledgments: We thank Kristin Kraus for editorial assistance.

Conflicts of Interest: The authors declare no conflict of interest.

\section{References}

1. Dang, L.; Su, S.-S.M. Isocitrate Dehydrogenase Mutation and (R)-2-Hydroxyglutarate: From Basic Discovery to Therapeutics Development. Annu. Rev. Biochem. 2017, 86, 305-331. [CrossRef] [PubMed]

2. Cairns, R.A.; Mak, T.W. Oncogenic Isocitrate Dehydrogenase Mutations: Mechanisms, Models, and Clinical Opportunities. Cancer Discov. 2013, 3, 730-741. [CrossRef] [PubMed]

3. Tommasini-Ghelfi, S.; Murnan, K.; Kouri, F.M.; Mahajan, A.S.; May, J.L.; Stegh, A.H. Cancer-Associated Mutation and beyond: The Emerging Biology of Isocitrate Dehydrogenases in Human Disease. Sci. Adv. 2019, 5, eaaw4543. [CrossRef]

4. Faubert, B.; Solmonson, A.; De Berardinis, R.J. Metabolic Reprogramming and Cancer Progression. Science 2020, 368 , eaaw5473. [CrossRef] [PubMed]

5. Han, S.; Liu, Y.; Cai, S.J.; Qian, M.; Ding, J.; Larion, M.; Gilbert, M.R.; Yang, C. IDH Mutation in Glioma: Molecular Mechanisms and Potential Therapeutic Targets. Br. J. Cancer 2020, 122, 1580-1589. [CrossRef]

6. Metallo, C.M.; Gameiro, P.A.; Bell, E.L.; Mattaini, K.R.; Yang, J.; Hiller, K.; Jewell, C.M.; Johnson, Z.R.; Irvine, D.J.; Guarente, L.; et al. Reductive Glutamine Metabolism by IDH1 Mediates Lipogenesis under Hypoxia. Nature 2012, 481, 380-384. [CrossRef]

7. Jiang, L.; Shestov, A.A.; Swain, P.; Yang, C.; Parker, S.J.; Wang, Q.A.; Terada, L.S.; Adams, N.D.; McCabe, M.T.; Pietrak, B.; et al. Reductive Carboxylation Supports Redox Homeostasis during Anchorage-Independent Growth. Nature 2016, 532, $255-258$. [CrossRef] [PubMed]

8. Itsumi, M.; Inoue, S.; Elia, A.J.; Murakami, K.; Sasaki, M.; Lind, E.F.; Brenner, D.; Harris, I.S.; Chio, I.I.C.; Afzal, S.; et al. Idh1 Protects Murine Hepatocytes from Endotoxin-Induced Oxidative Stress by Regulating the Intracellular NADP+/NADPH Ratio. Cell Death Differ. 2015, 22, 1837-1845. [CrossRef]

9. Parsons, D.W.; Jones, S.; Zhang, X.; Lin, J.C.-H.; Leary, R.J.; Angenendt, P.; Mankoo, P.; Carter, H.; Siu, I.-M.; Gallia, G.L.; et al. An Integrated Genomic Analysis of Human Glioblastoma Multiforme. Science 2008, 321, 1807-1812. [CrossRef]

10. Balss, J.; Meyer, J.; Mueller, W.; Korshunov, A.; Hartmann, C.; Deimling, A. von Analysis of the IDH1 Codon 132 Mutation in Brain Tumors. Acta Neuropathol. 2008, 116, 597-602. [CrossRef] 
11. Yan, H.; Parsons, D.W.; Jin, G.; McLendon, R.; Rasheed, B.A.; Yuan, W.; Kos, I.; Batinic-Haberle, I.; Jones, S.; Riggins, G.J.; et al. IDH1 and IDH2 Mutations in Gliomas. N. Engl. J. Med. 2009, 360, 765-773. [CrossRef]

12. Pusch, S.; Schweizer, L.; Beck, A.-C.; Lehmler, J.-M.; Weissert, S.; Balss, J.; Miller, A.K.; Deimling, A. von D-2-Hydroxyglutarate Producing Neo-Enzymatic Activity Inversely Correlates with Frequency of the Type of Isocitrate Dehydrogenase 1 Mutations Found in Glioma. Acta Neuropathol. Commun. 2014, 2, 19. [CrossRef] [PubMed]

13. Huang, L.E. Friend or Foe-IDH1 Mutations in Glioma 10 Years On. Carcinogenesis 2019, 40, 1299-1307. [CrossRef]

14. Bleeker, F.E.; Lamba, S.; Leenstra, S.; Troost, D.; Hulsebos, T.; Vandertop, W.P.; Frattini, M.; Molinari, F.; Knowles, M.; Cerrato, A.; et al. IDH1 Mutations at Residue p.R132 (IDH1R132) Occur Frequently in High-grade Gliomas but Not in Other Solid Tumors. Hum. Mutat. 2009, 30, 7-11. [CrossRef]

15. Dang, L.; Jin, S.; Su, S.M. IDH Mutations in Glioma and Acute Myeloid Leukemia. Trends Mol. Med. 2010, 16, 387-397. [CrossRef]

16. Dang, L.; White, D.W.; Gross, S.; Bennett, B.D.; Bittinger, M.A.; Driggers, E.M.; Fantin, V.R.; Jang, H.G.; Jin, S.; Keenan, M.C.; et al. Cancer-Associated IDH1 Mutations Produce 2-Hydroxyglutarate. Nature 2009, 462, 739-744. [CrossRef]

17. Xu, W.; Yang, H.; Liu, Y.; Yang, Y.; Wang, P.; Kim, S.-H.; Ito, S.; Yang, C.; Wang, P.; Xiao, M.-T.; et al. Oncometabolite 2Hydroxyglutarate Is a Competitive Inhibitor of $\alpha$-Ketoglutarate-Dependent Dioxygenases. Cancer Cell 2011, 19, 17-30. [CrossRef] [PubMed]

18. Lu, C.; Ward, P.S.; Kapoor, G.S.; Rohle, D.; Turcan, S.; Abdel-Wahab, O.; Edwards, C.R.; Khanin, R.; Figueroa, M.E.; Melnick, A.; et al. IDH Mutation Impairs Histone Demethylation and Results in a Block to Cell Differentiation. Nature 2012, 483, 474-478. [CrossRef] [PubMed]

19. Turcan, S.; Rohle, D.; Goenka, A.; Walsh, L.A.; Fang, F.; Yilmaz, E.; Campos, C.; Fabius, A.W.M.; Lu, C.; Ward, P.S.; et al. IDH1 Mutation Is Sufficient to Establish the Glioma Hypermethylator Phenotype. Nature 2012, 483, 479-483. [CrossRef] [PubMed]

20. Tiburcio, P.D.B.; Locke, M.C.; Bhaskara, S.; Chandrasekharan, M.B.; Huang, L.E. The Neural Stem-Cell Marker CD24 Is Specifically Upregulated in IDH-Mutant Glioma. Transl. Oncol. 2020, 13, 100819. [CrossRef]

21. Losman, J.-A.; Kaelin, W.G. What a Difference a Hydroxyl Makes: Mutant IDH, (R)-2-Hydroxyglutarate, and Cancer. Gene Dev. 2013, 27, 836-852. [CrossRef]

22. Miller, J.J.; Shih, H.A.; Andronesi, O.C.; Cahill, D.P. Isocitrate Dehydrogenase-mutant Glioma: Evolving Clinical and Therapeutic Implications. Cancer 2017, 123, 4535-4546. [CrossRef] [PubMed]

23. Watanabe, T.; Nobusawa, S.; Kleihues, P.; Ohgaki, H. IDH1 Mutations Are Early Events in the Development of Astrocytomas and Oligodendrogliomas. Am. J. Pathol. 2009, 174, 1149-1153. [CrossRef]

24. Network, C.G.A.R.; Brat, D.J.; Verhaak, R.G.W.; Aldape, K.D.; Yung, W.K.A.; Salama, S.R.; Cooper, L.A.D.; Rheinbay, E.; Miller, C.R.; Vitucci, M.; et al. Comprehensive, Integrative Genomic Analysis of Diffuse Lower-Grade Gliomas. N. Engl. J. Med. 2015, 372, 2481-2498. [CrossRef]

25. Kaiser, A.M.; Attardi, L.D. Deconstructing Networks of P53-Mediated Tumor Suppression in Vivo. Cell Death Differ. 2018, 25, 93-103. [CrossRef]

26. Sabapathy, K.; Lane, D.P. Therapeutic Targeting of P53: All Mutants Are Equal, but Some Mutants Are More Equal than Others. Nat. Rev. Clin. Oncol. 2018, 15, 13-30. [CrossRef]

27. Boutelle, A.M.; Attardi, L.D. P53 and Tumor Suppression: It Takes a Network. Trends Cell Biol. 2021. [CrossRef]

28. Cerami, E.; Gao, J.; Dogrusoz, U.; Gross, B.E.; Sumer, S.O.; Aksoy, B.A.; Jacobsen, A.; Byrne, C.J.; Heuer, M.L.; Larsson, E.; et al. The CBio Cancer Genomics Portal: An Open Platform for Exploring Multidimensional Cancer Genomics Data. Cancer Discov. 2012, 2, 401-404. [CrossRef]

29. Gao, J.; Aksoy, B.A.; Dogrusoz, U.; Dresdner, G.; Gross, B.; Sumer, S.O.; Sun, Y.; Jacobsen, A.; Sinha, R.; Larsson, E.; et al. Integrative Analysis of Complex Cancer Genomics and Clinical Profiles Using the CBioPortal. Sci. Signal. 2013, 6, pl1. [CrossRef]

30. Huang, L.E.; Cohen, A.L.; Colman, H.; Jensen, R.L.; Fults, D.W.; Couldwell, W.T. IGFBP2 Expression Predicts IDH-Mutant Glioma Patient Survival. Oncotarget 2016, 8, 191-202. [CrossRef] [PubMed]

31. Karsy, M.; Guan, J.; Huang, L.E. Prognostic Role of Mitochondrial Pyruvate Carrier in Isocitrate Dehydrogenase-Mutant Glioma. J. Neurosurg. 2018, 130, 56-66. [CrossRef]

32. Sanson, M.; Marie, Y.; Paris, S.; Idbaih, A.; Laffaire, J.; Ducray, F.; Hallani, S.E.; Boisselier, B.; Mokhtari, K.; Hoang-Xuan, K.; et al. Isocitrate Dehydrogenase 1 Codon 132 Mutation Is an Important Prognostic Biomarker in Gliomas. J. Clin. Oncol. 2009, 27, 4150-4154. [CrossRef] [PubMed]

33. Tesileanu, C.M.S.; Vallentgoed, W.R.; Sanson, M.; Taal, W.; Clement, P.M.; Wick, W.; Brandes, A.A.; Baurain, J.F.; Chinot, O.L.; Wheeler, H.; et al. Non-IDH1-R132H IDH1/2 Mutations Are Associated with Increased DNA Methylation and Improved Survival in Astrocytomas, Compared to IDH1-R132H Mutations. Acta Neuropathol. 2021, 141, 945-957. [CrossRef] [PubMed]

34. Castro, L.N.G.; Wesseling, P. The CIMPACT-NOW Updates and Their Significance to Current Neuro-Oncology Practice. NeuroOncol. Pract. 2020, 8, 4-10. [CrossRef]

35. Harris, C.C.; Hollstein, M. Clinical Implications of the P53 Tumor-Suppressor Gene. N. Engl J. Med. 1993, 329, 1318-1327. [CrossRef]

36. Muller, P.A.J.; Vousden, K.H. P53 Mutations in Cancer. Nat. Cell Biol. 2013, 15, 2-8. [CrossRef] [PubMed]

37. Shen, D.; Zhang, J.; Yuan, K.; Zhao, J.; Zhao, Z.; Cui, L.; Zhang, Y.; Wang, G.; Cai, S.; Bai, Y.; et al. Landscape of IDH1/2 Mutations in Chinese Patients with Solid Tumors: A Pan-cancer Analysis. Mol. Genet. Genom. Med. 2021, e1697. [CrossRef] 
38. Zheng, S.; Alfaro-Munoz, K.; Wei, W.; Wang, X.; Wang, F.; Eterovic, A.K.; Shaw, K.R.M.; Meric-Bernstam, F.; Fuller, G.N.; Chen, K.; et al. Prospective Clinical Sequencing of Adult Glioma. Mol. Cancer Ther. 2019, 18, 991-1000. [CrossRef]

39. Ward, P.S.; Lu, C.; Cross, J.R.; Abdel-Wahab, O.; Levine, R.L.; Schwartz, G.K.; Thompson, C.B. The Potential for Isocitrate Dehydrogenase Mutations to Produce 2-Hydroxyglutarate Depends on Allele Specificity and Subcellular Compartmentalization. J. Biol. Chem. 2013, 288, 3804-3815. [CrossRef]

40. Wang, T.-X.; Liang, J.-Y.; Zhang, C.; Xiong, Y.; Guan, K.-L.; Yuan, H.-X. The Oncometabolite 2-Hydroxyglutarate Produced by Mutant IDH1 Sensitizes Cells to Ferroptosis. Cell Death Dis. 2019, 10, 755. [CrossRef]

41. Stockwell, B.R.; Angeli, J.P.F.; Bayir, H.; Bush, A.I.; Conrad, M.; Dixon, S.J.; Fulda, S.; Gascón, S.; Hatzios, S.K.; Kagan, V.E.; et al. Ferroptosis: A Regulated Cell Death Nexus Linking Metabolism, Redox Biology, and Disease. Cell 2017, 171, 273-285. [CrossRef]

42. Qing, Y.; Dong, L.; Gao, L.; Li, C.; Li, Y.; Han, L.; Prince, E.; Tan, B.; Deng, X.; Wetzel, C.; et al. R-2-Hydroxyglutarate Attenuates Aerobic Glycolysis in Leukemia by Targeting the FTO/M6A/PFKP/LDHB Axis. Mol. Cell 2021, 81, 922-939.E9. [CrossRef]

43. Tiburcio, P.D.B.; Xiao, B.; Berg, S.; Asper, S.; Lyne, S.; Zhang, Y.; Zhu, X.; Yan, H.; Huang, L.E. Functional Requirement of a Wild-Type Allele for Mutant IDH1 to Suppress Anchorage-Independent Growth through Redox Homeostasis. Acta Neuropathol. 2018, 135, 285-298. [CrossRef]

44. Tiburcio, P.D.B.; Xiao, B.; Chai, Y.; Asper, S.; Tripp, S.R.; Gillespie, D.L.; Jensen, R.L.; Huang, L.E. IDH1R132H Is Intrinsically Tumor-Suppressive but Functionally Attenuated by the Glutamate-Rich Cerebral Environment. Oncotarget 2018, 9, 35100-35113. [CrossRef]

45. Schneider, G.; Schmidt-Supprian, M.; Rad, R.; Saur, D. Tissue-Specific Tumorigenesis: Context Matters. Nat. Rev. Cancer 2017, 17, 239-253. [CrossRef]

46. Haigis, K.M.; Cichowski, K.; Elledge, S.J. Tissue-Specificity in Cancer: The Rule, Not the Exception. Science 2019, 363, 1150-1151. [CrossRef]

47. Amankulor, N.M.; Kim, Y.; Arora, S.; Kargl, J.; Szulzewsky, F.; Hanke, M.; Margineantu, D.H.; Rao, A.; Bolouri, H.; Delrow, J.; et al. Mutant IDH1 Regulates the Tumor-Associated Immune System in Gliomas. Genes Dev. 2017, 31, 774-786. [CrossRef]

48. Pirozzi, C.J.; Carpenter, A.B.; Waitkus, M.S.; Wang, C.Y.; Zhu, H.; Hansen, L.J.; Chen, L.H.; Greer, P.K.; Feng, J.; Wang, Y.; et al. Mutant IDH1 Disrupts the Mouse Subventricular Zone and Alters Brain Tumor Progression. Mol. Cancer Res. 2017, 15, 507-520. [CrossRef]

49. Núñez, F.J.; Mendez, F.M.; Kadiyala, P.; Alghamri, M.S.; Savelieff, M.G.; Garcia-Fabiani, M.B.; Haase, S.; Koschmann, C.; Calinescu, A.-A.; Kamran, N.; et al. IDH1-R132H Acts as a Tumor Suppressor in Glioma via Epigenetic up-Regulation of the DNA Damage Response. Sci. Transl. Med. 2019, 11, eaaq1427. [CrossRef]

50. Jiang, P.; Du, W.; Wang, X.; Mancuso, A.; Gao, X.; Wu, M.; Yang, X. P53 Regulates Biosynthesis through Direct Inactivation of Glucose-6-Phosphate Dehydrogenase. Nat. Cell Biol. 2011, 13, 310-316. [CrossRef]

51. Li, T.; Kon, N.; Jiang, L.; Tan, M.; Ludwig, T.; Zhao, Y.; Baer, R.; Gu, W. Tumor Suppression in the Absence of P53-Mediated Cell-Cycle Arrest, Apoptosis, and Senescence. Cell 2012, 149, 1269-1283. [CrossRef]

52. Jiang, L.; Kon, N.; Li, T.; Wang, S.-J.; Su, T.; Hibshoosh, H.; Baer, R.; Gu, W. Ferroptosis as a P53-Mediated Activity during Tumour Suppression. Nature 2015, 520, 57-62. [CrossRef]

53. Ou, Y.; Wang, S.-J.; Li, D.; Chu, B.; Gu, W. Activation of SAT1 Engages Polyamine Metabolism with P53-Mediated Ferroptotic Responses. Proc. Natl. Acad. Sci. USA 2016, 113, E6806-E6812. [CrossRef]

54. Wang, S.-J.; Li, D.; Ou, Y.; Jiang, L.; Chen, Y.; Zhao, Y.; Gu, W. Acetylation Is Crucial for P53-Mediated Ferroptosis and Tumor Suppression. Cell Rep. 2016, 17, 366-373. [CrossRef]

55. Yang, H.; Zhao, L.; Gao, Y.; Yao, F.; Marti, T.M.; Schmid, R.A.; Peng, R.-W. Pharmacotranscriptomic Analysis Reveals Novel Drugs and Gene Networks Regulating Ferroptosis in Cancer. Cancers 2020, 12, 3273. [CrossRef]

56. Tiburcio, P.D.B.; Gillespie, D.L.; Jensen, R.L.; Huang, L.E. Extracellular Glutamate and IDH1R132H Inhibitor Promote Glioma Growth by Boosting Redox Potential. J. Neuro-Oncol. 2020, 146, 427-437. [CrossRef]

57. McLendon, R.; Friedman, A.; Bigner, D.; Meir, E.G.V.; Brat, D.J.; Mastrogianakis, G.M.; Olson, J.J.; Mikkelsen, T.; Lehman, N.; Aldape, K.; et al. Comprehensive Genomic Characterization Defines Human Glioblastoma Genes and Core Pathways. Nature 2008, 455, 1061-1068. [CrossRef]

58. Mukasa, A.; Takayanagi, S.; Saito, K.; Shibahara, J.; Tabei, Y.; Furuya, K.; Ide, T.; Narita, Y.; Nishikawa, R.; Ueki, K.; et al. Significance of IDH Mutations Varies with Tumor Histology, Grade, and Genetics in Japanese Glioma Patients. Cancer Sci. 2012, 103, 587-592. [CrossRef]

59. Wang, X.-W.; Ciccarino, P.; Rossetto, M.; Boisselier, B.; Marie, Y.; Desestret, V.; Gleize, V.; Mokhtari, K.; Sanson, M.; Labussière, M. IDH Mutations: Genotype-Phenotype Correlation and Prognostic Impact. Biomed Res. Int. 2014, 2014, 540236. [CrossRef]

60. Dong, C.; Yuan, Z.; Li, Q.; Wang, Y. The Clinicopathological and Prognostic Significance of TP53 Alteration in K27M Mutated Gliomas: An Individual-Participant Data Meta-Analysis. Neurol. Sci. 2018, 39, 1191-1201. [CrossRef]

61. Yang, P.; Cai, J.; Yan, W.; Zhang, W.; Wang, Y.; Chen, B.; Li, G.; Li, S.; Wu, C.; Yao, K.; et al. Classification Based on Mutations of TERT Promoter and IDH Characterizes Subtypes in Grade II/III Gliomas. Neuro-Oncology 2016, 18, 1099-1108. [CrossRef]

62. Da, R.; Wang, M.; Jiang, H.; Wang, T.; Wang, W. BRAF AMP Frequently Co-Occurs with IDH1/2, TP53, and ATRX Mutations in Adult Patients with Gliomas and Is Associated with Poorer Survival Than That of Patients Harboring BRAF V600E. Front. Oncol. 2021, 10, 531968. [CrossRef] [PubMed] 